OPEN ACCESS

Edited by:

Peizeng Yang,

First Affiliated Hospital of Chongqing Medical University, China

Reviewed by:

Yohei Tomita,

Boston Children's Hospital and Harvard Medical School, United States

Kuender D. Yang,

Mackay Memorial Hospital, Taiwan

*Correspondence: Blanca Molins bmolins@clinic.cat

Specialty section: This article was submitted to Autoimmune and Autoinflammatory Disorders, a section of the journal

Frontiers in Immunology

Received: 01 July 2020 Accepted: 14 September 2020 Published: 25 September 2020

Citation:

Matas J, Llorenç V, Fonollosa A, Díaz-Valle D, Esquinas $C$, de la Maza MTS, Figueras-Roca M, Artaraz J, Berasategui B, Mesquida M, Adán A and Molins B (2020) Systemic Regulatory T Cells and IL-6 as Prognostic Factors for

Anatomical Improvement of Uveitic Macular Edema.

Front. Immunol. 11:579005. doi: 10.3389/fimmu.2020.579005

\section{Systemic Regulatory T Cells and IL-6 as Prognostic Factors for Anatomical Improvement of Uveitic Macular Edema}

\author{
Jessica Matas ${ }^{1}$, Victor Llorenç ${ }^{1}$, Alex Fonollosa ${ }^{2}$, David Díaz-Valle ${ }^{3}$, Cristina Esquinas ${ }^{4}$, \\ Maria Teresa Sainz de la Maza ${ }^{1}$, Marc Figueras-Roca ${ }^{1}$, Joseba Artaraz ${ }^{2}$, \\ Barbara Berasategui ${ }^{2}$, Marina Mesquida ${ }^{1}$, Alfredo Adán $^{1}$ and Blanca Molins ${ }^{1 \star}$ \\ ${ }^{1}$ Group of Ocular Inflammation, Institut d'Investigacions Biomèdiques Agustí Pi i Sunyer (IDIBAPS), Hospital Clínic de \\ Barcelona, Barcelona, Spain, ${ }^{2}$ Department of Ophthalmology, BioCruces Health Research Institute, Hospital Cruces, \\ University of the Basque Country, Baracaldo, Spain, ${ }^{3}$ Ophthalmology Department and Health Research Institute (IdISSC), \\ Hospital Clinic of San Carlos, Madrid, Spain, ${ }^{4}$ Valle Hebron Research Institute, Autonomous University of Barcelona, \\ Barcelona, Spain
}

Purpose: To investigate whether systemic immune mediators and circulating regulatory $T$ cells (Tregs) could be prognostic factors for anatomic outcomes in macular edema secondary to non-infectious uveitis (UME).

Methods: Multicenter, prospective, observational, 12-month follow-up study of 60 patients with UME. Macular edema was defined as central subfield thickness (CST) > $300 \mu \mathrm{m}$ measured with spectral domain optical coherence tomography (SD-OCT). Serum samples and peripheral blood mononuclear cells (PBMC) were obtained from venous blood extraction at baseline. Serum levels of IL-1 $\beta$, IL-6, IL-8, IL-17, MCP-1, TNF- $\alpha$, IL-10, and VEGF were determined by Luminex. Tregs population, defined as $\mathrm{CD} 3^{+} \mathrm{CD} 4^{+} \mathrm{FoxP} 3^{+}$ in PBMC, was determined by flow cytometry. Main outcome measure was the predictive association between searched mediators and CST sustained improvement, defined as CST $<300$ microns or a 20\% CST decrease, at 6 months maintained until 12-months compared to baseline levels.

Results: Multivariate logistic regression analysis showed an association between CST sustained improvement at 12 months follow-up and IL-6 and Tregs baseline levels. Higher IL-6 levels were associated with less events of UME improvement (OR: 0.67, 95\% Cl (0.45-1.00), $\mathrm{P}=0.042)$, whereas higher levels of Tregs favored such improvement (OR: 1.25, 95\% Cl: 1.12-2.56, $\mathrm{P}=0.049$ ).

Conclusions: Increased levels of Tregs and reduced levels of IL-6 in serum may be prognostic factors of sustained anatomical improvement in UME. These findings could enforce the opportunity to develop more efficient and personalized therapeutic approaches to improve long-term visual prognosis in patients with UME.

Keywords: macular edema, uveitis, cytokines, regulatory T cells, biomarkers 


\section{INTRODUCTION}

Non-infectious uveitis accounts for $10 \%$ of cases of total blindness in developed world and may result in persistent visual impairment in untreated patients (1). Uveitis may affect people of all ages but most frequently occurs in the working age population, thus implying a high socioeconomical burden (2). Macular edema remains the most commonly found structural complication of uveitis that results in central visual impairment although only one third of uveitis patients develop it (3). The pathogenesis of uvetitic macular edema (UME) involves breakdown of the blood-retinal barrier, followed by both intracellular and extracellular fluid accumulation within the macular retina (4). However, the underlying pathophysiology as well as the molecular mediators involved are not fully understood, therefore making UME a controversial topic.

Chronic retinal inflammation has been observed in early phases and also in the sight-threatening advanced forms of UME. The uveitic retina is characterized by a complex milieu of dysregulated proinflammatory factors. Increased levels of certain proinflammatory cytokines, such as interleukin-6 (IL-6), IL-8, and tumor necrosis factor- $\alpha$ (TNF- $\alpha$ ), both in serum and aqueous humor, have been found in patients with UME (5). Vascular endothelial growth factor (VEGF) may also play a major role as it can be increased in aqueous humor of patients with UME and in other forms of macular edema $(5,6)$. On the other hand, in the last years, the interest in regulatory $\mathrm{T}$ cells (Tregs) as markers of activity and modulators of autoreactive $\mathrm{T}$ lymphocytes in uveitis and other inflammatory diseases has gained attention (7). Patients with active uveitis have reduced circulating levels of Tregs compared to healthy subjects, with Tregs levels being significantly upregulated during quiescent disease $(8,9)$. Moreover, adoptive transfer of Tregs has resulted in greater protection against intraocular inflammation in experimental models of uveitis $(10,11)$. However, it is unknown whether Tregs are also involved in the pathophysiology of UME.

UME may persist despite adequate control of activity, thus leading to permanent photoreceptor damage and loss of central visual acuity (12). After the acute phase, UME may either subside spontaneously or respond to treatment intended to reduce local inflammatory mediators. Moreover, in relapsing and chronic forms of UME, especially in bilateral forms, systemic treatment should be started, usually consisting of corticosteroids and/or immunomodulators (13). However, despite these options, a number of patients still remain refractory to treatment and UME may lead to severe visual impairment.

In summary, there is scarce data regarding the factors influencing sustained anatomic and visual recovery in eyes with UME. A better understanding of the prognostic biomarkers in patients with UME would allow the use of more efficient and selective pharmacological approaches to improve long-term visual prognosis and minimizing unnecessary druginduced toxicity. In this prospective study, we aimed to investigate the association of several systemic immune mediators and Tregs with the anatomic outcome of UME after 12 months of follow-up.

\section{MATERIALS AND METHODS}

\section{Study Design and Selection Criteria}

We conducted a multicenter, prospective, observational, 12month follow-up study to analyze the association of peripheral blood immune mediators and Tregs with the clinical evolution of UME. Adult ( $>18$ years-old) patients with non-infectious UME in at least one eye were proposed for inclusion. UME was defined as central subfield thickness (CST) of $>300 \mu \mathrm{m}$ as measured by SDOCT (HD-Cirrus, Carl Zeiss Meditec, Dublin, CA) and presence of fluid (intraretinal and/or subretinal) in the macula. Whenever bilateral UME was present, the eye with the higher CST value in the fovea according to OCT measurements in $\mu \mathrm{m}$ was selected as the study eye. Exclusion criteria were limited to infectious uveitis, highly hazy ocular media, concurrent ocular diseases, exclusive tractional UME, pregnancy, immunocompromising systemic diseases (including, but not limited to HIV, leukemia, lymphoma, and chemotherapy), and eyes with any intervention (intraocular surgery, laser, and intravitreal therapy) in the previous 4 months.

Three referral centers for ocular inflammatory diseases in Spain (Clínic Hospital of Barcelona, Cruces Hospital of Bilbao and Clínico San Carlos Hospital of Madrid) participated in the recruitment of patients from January 2015 to January 2017. Local ethics committees approved the study (Comité Ético de Investigación Clínica del Hospital Clínic de Barcelona 2013/ 8574; Comité de Ética de la Investigación con Medicamentos de Euskadi, Hospital Universitario Cruces PI201406; Comité Ético de Investigación Clínica del Hospital Clínico San Carlos de Madrid 13/244-E). All patients provided written informed consent, and the research followed the regulations of the Declaration of Helsinki.

\section{Data Collection and Ophthalmic Assessment}

Patients were evaluated at each clinical site, and protocol-based assessments were performed at different time-points: baseline, month 1 , month 6 , and month 12 of follow-up. Other visits at different time-points were allowed at the discretion of the treating physician. Data were recorded in an electronic case report form designed ad hoc.

Medical record data from each patient included demographics (age, race, and sex), diagnosis classified by anatomic location according to the Standardization of Uveitis Nomenclature (SUN) criteria (14), laterality of disease, systemic or isolated ocular inflammatory disease classification, and a complete ophthalmic examination.

Data gathered from evaluation of each patient included bestcorrected visual acuity (BCVA, with Snellen charts in decimal scale at a test distance of $6 \mathrm{~m}$ ), the presence or absence of disease activity, categorical and quantitative OCT findings, and UME treatment. The SUN recommendations were used for anterior chamber and vitreous inflammatory activity gradations. SDOCT exploration was used as determination of UME using Macular cube $512 \times 128$ A-scan, within a $6 \times 6 \mathrm{~mm}$ area centered on the fovea. Imaging assessment was performed by 
two investigators who were masked to clinical data of the corresponding patients. In the event of discrepancies, the two graders made the assessment together and reached a consensus. Masked investigators were asked to determine the CST and the pattern of UME (sub-retinal fluid, cystoid, diffuse, or tractional components). Macular volume, subfoveal choroidal thickness (enhanced deep imaging mode), maximum diameter of the greatest cyst if present, subretinal fluid pocket's length in a transfoveal B-scan were also recorded. Ancillary tests to rule out infectious origin (including but not limited to luetic serology, quantiFERON-TB Gold and toxoplasma serology) and to appropriately classify the uveitis were ordered at the investigator discretion.

\section{Sample Collection}

Two peripheral blood samples were obtained at the time of patient inclusion in the presence of UME according to the aforementioned definition. Serum was obtained from sample 1 as follows: blood was centrifugated at $1600 \mathrm{~g}$ within $20 \mathrm{~min}$ and stored at $-80^{\circ} \mathrm{C}$ until inflammatory mediators were determined. The levels of Tregs in peripheral blood were determined from sample 2: peripheral blood mononuclear cells (PBMCs) were obtained by Ficoll (Ficoll-Plaque Plus, GE Healthcare) gradient centrifugation, washed twice with RPMI 1640 containing 2\% heat-inactivated fetal calf serum and cryopreserved for further staining and analysis by flow cytometry. Cryopreserved serum and PBMC samples were then shipped to the Ocular Inflammation Laboratory (Hospital Clinic of Barcelona) where they were analyzed.

\section{Cytokine Determination}

Eight immune mediators were determined: IL-1 $\beta$, IL-6, IL-8, IL17, MCP-1, TNF- $\alpha$, IL-10, and VEGF. These molecules were chosen based on published results of previous studies regarding both local and systemic biomarkers in uveitis and UME. Selected immune mediators were determined by a Luminex platform (Millipore's MilliPlex Human Cytokine/Chemokine kit) used to measure cytokine and chemokine levels in serum samples using an assay plate layout consisting of seven standards in duplicate (3.2-2,000 pg/mL), one blank well (for background fluorescence subtraction), two internal quality control samples in duplicate, and $25-\mu \mathrm{L}$ duplicates of each serum sample. The MilliPlex method was performed as recommended by the manufacturer. Zero values were statistically handled as a third of the provided detection limit.

\section{Treg Determination by Flow Cytometry}

FoxP3 levels in $\mathrm{CD}^{+} \mathrm{CD}^{+}$cells from cryopreserved PBMCs were determined by flow cytometry. A live/dead Fixable Dead Cell Stain (Invitrogen) was used to exclude dead cells from the analysis, followed by incubation for $30 \mathrm{~min}$ on ice with AF700-CD3 (clone OKT3) and FITC-CD4 (OKT4) (eBiosciences). Cells were then washed, fixed and permeabilized before adding PE-FOXP3 (clone 236A/E7) (eBiosciences) for $30 \mathrm{~min}$. Appropriate isotype controls were included in each experiment. Flow cytometric analyses were performed on a Fortessa flow cytometer (BD Biosciences) with a total of 50,000 events recorded for each sample through a live $\mathrm{CD}^{+} \mathrm{CD}^{+}$lymphocyte gate. Analysis of flow cytometry data was performed using FlowJo software. Tregs were defined as $\mathrm{CD}^{+} \mathrm{CD}^{+} \mathrm{FoxP}^{+}$cells.

\section{Outcome Measures}

To determine whether Tregs and serum immune mediators can be predictive factors for anatomic outcomes in UME, we analyzed the association between baseline levels of the abovementioned immune mediators and Tregs with sustained anatomical improvement of UME. Improvement of UME was defined as a $20 \%$ CST decrease or CST $<300 \mu \mathrm{m}$ at 6 months and maintained at 12 months of follow-up compared to baseline.

\section{Statistical Analysis}

To describe the qualitative variables, absolute frequencies and percentages were used. The description of quantitative variables was performed using the mean and standard deviation (SD). The Kolmogorov-Smirnov test was used to assess the normality of distributions. In the case of quantitative variables, the comparison of the characteristics of the eyes depending on the presence of sustained anatomical improvement was carried out using the Student t-test (or Mann-Whitney U-test if normality was not assumed). The Chi-squared test (Fisher test for frequencies < 5) was used for the comparison of categorical variables. Wilcoxon test was performed in order to analyze changes in CST during follow up.

A back stepwise logistic regression analysis was developed including the defined and CST-based favorable anatomical outcome item as the dependent variable, and as independent variables, those cytokines and clinical variables with a $\mathrm{P}$-value < 0.2 in the univariate analysis and clinical pertinence (evolution time of the EMU to baseline, age at baseline, and sex). The results have been described with odds ratios (OR), 95\% confidence intervals $(\mathrm{CI})$, and $\mathrm{P}$-values. Linear relationships between defined biomarkers were assessed using Spearman tests. For all tests, values of $\mathrm{P}<0.05$ were considered statistically significant. The R Studio statistical package (version 2.5) was used for statistical analysis.

\section{RESULTS}

Sixty eyes of sixty patients were included. The mean age of the group was 51.1 years (SD, 15.1; range, 21-89). The mean previous duration of UME at the inclusion time was 17.37 months (SD, 31.5; range, 0-144 months). Table 1 shows patients' demographics and clinical data. The description of etiological diagnosis showed that systemic disorders were the predominant ones $(n=24)$, followed by isolated ocular syndromes $(\mathrm{n}=21)$, and unclassified uveitis $(\mathrm{n}=15)$.

The overall mean CST was $437.8 \pm 122.2$ (SD) $\mu \mathrm{m}$ at baseline and decreased significantly after 1 month to $357.4 \pm 118.9 \mu \mathrm{m}$ $(\mathrm{P}<0.001)$, reaching $337.73 \pm 135.5 \mu \mathrm{m}$ at 6 months and $329.6 \pm$ $108.1 \mu \mathrm{m}$ at 12 months $(\mathrm{P}<0.001)$. Reduction in CST followed a similar pattern in systemically treated patients and non- 
TABLE 1 | Baseline ophthalmic characteristics in eyes with uveitic macular edema.

\section{Baseline characteristics}

\begin{tabular}{|c|c|}
\hline Eyes/Patients (n/n) & $60 / 60$ \\
\hline Evolution time to baseline (months, mean $\pm \mathrm{SD}$ ) & $17 \pm 31$ \\
\hline Follow-up (months) & 12 \\
\hline Age (years, mean $\pm \mathrm{SD}$ ) & $51 \pm 15$ \\
\hline Female, n (\%) & $35(58.3)$ \\
\hline Bilateral, n (\%) & $23(38.3)$ \\
\hline \multicolumn{2}{|l|}{ Patterns of $\mathrm{UME}^{1}$} \\
\hline Cystoid, n (\%) & $47(78.3)$ \\
\hline Diffuse, n (\%) & $14(23.3)$ \\
\hline Subretinal fluid, n (\%) & $21(35)$ \\
\hline Tractional component ${ }^{2}, \mathrm{n}(\%)$ & $18(30)$ \\
\hline \multicolumn{2}{|l|}{ Anatomical classification (SUN) } \\
\hline Anterior, n (\%) & $16(26.6)$ \\
\hline Intermediate, n (\%) & $9(15)$ \\
\hline Posterior, n (\%) & $22(36.7)$ \\
\hline Panuveitis, n (\%) & $13(21.7)$ \\
\hline \multicolumn{2}{|l|}{ Etiologic classification } \\
\hline Unclassifiable (undifferentiated), n (\%) & $15(25)$ \\
\hline Seronegative spondyloarthropathies, n (\%) & $12(20)$ \\
\hline Ankylosing spondylitis, $n$ & 6 \\
\hline HLA-B27 + (without SA), n & 3 \\
\hline Inflammatory bowel disease, $\mathrm{n}$ & 1 \\
\hline Psoriatic arthritis, $\mathrm{n}$ & 1 \\
\hline Reactive arthritis, $\mathrm{n}$ & 1 \\
\hline White dot syndromes,n (\%) & $14(23.3)$ \\
\hline Birdshot, n & 13 \\
\hline Serpiginous, $n$ & 1 \\
\hline Other etiologies, n (\%) & 19 (31.6) \\
\hline Sarcoidosis, $\mathrm{n}$ & 6 \\
\hline Vogt-Koyanagi-Harada, n & 5 \\
\hline Juvenile idiopathic arthritis & 2 \\
\hline Multiple sclerosis, n & 2 \\
\hline Pars planitis, $\mathrm{n}$ & 2 \\
\hline Sympathetic ophthalmia, n & 1 \\
\hline IRVAN, n & 1 \\
\hline \multicolumn{2}{|l|}{ Treatment/s for UME ${ }^{1}$} \\
\hline Topical, n (\%) & $2(3.3)$ \\
\hline Local (only), n (\%) & 14(23.3) \\
\hline TCA (peri/intraocular), n & 5 \\
\hline Dexamethasone implant, $\mathrm{n}$ & 11 \\
\hline Systemic \pm local, n (\%) & $40(66.7)$ \\
\hline Oral Corticosteroids, $\mathrm{n}$ & 37 \\
\hline Classic Immunomodulators, $\mathrm{n}$ & 19 \\
\hline Biologics, n & 13 \\
\hline Previous vitrectomy, n (\%) & $2(3.3)$ \\
\hline
\end{tabular}

${ }^{1}$ One eye may belong to more than one category (four eyes did not receive any treatment). ${ }^{2}$ t refers to qualitatively thickened vitreo-macular interface.

UME, uveitis macular edema; SUN, standardization of uveitis nomenclature classification; SA, spondyloarthropaties; IRVAN, idiopathic retinal vasculitis, aneurisms and neuroretinitis syndrome; TCA, triamcinolone acetonide injections.

systemically treated eyes, reaching a significant reduction already at month 1 and maintaining a sustained reduction throughout the 12-month follow-up (Figure 1).

Cytokines and Tregs levels were compared between patients with and without systemic treatment. In our cohort, $66.7 \%$ of patients had received systemic treatment for their underlying disease (non-infectious uveitis), and $33.3 \%$ had not received systemic treatment for at least 6 months before inclusion. There were no significant differences in the levels of cytokines and Tregs between both groups (Table 2). However, differences among specific treatments could not properly be evaluated due to the reduced sample size in each specific treatment subgroup.
In the univariate analysis, qualitatively assessed significant tractional component of the UME was associated to nonsustained anatomical improvement, whereas presence of subretinal fluid was associated to sustained improvement in UME (Table 3). To evaluate prognostic factors for UME outcome, variables were analyzed according to the pre-defined CST improvement event with a Step Backwise Logistic Regression Model. Higher macular volume at baseline was the only clinical variable that precluded favorable UME outcome (OR: 1.41, 95\% CI: 0.97-2.03, P = 0.032). Interestingly, higher baseline levels of IL- 6 were associated with no sustained anatomical improvement of UME at 12 months of follow-up 

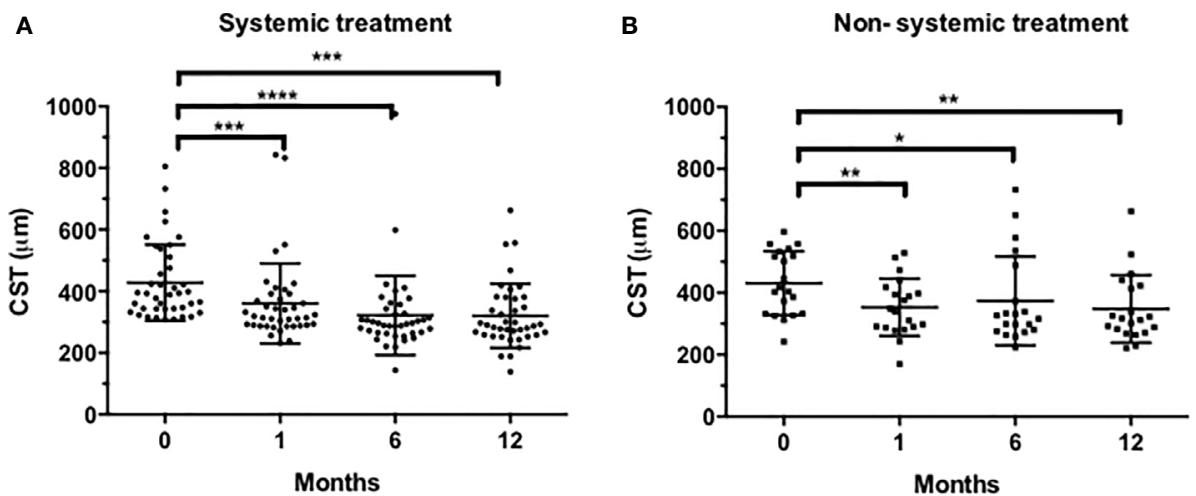

FIGURE 1 | Evolution of central subfield thickness (CST) in systemically treated eyes (A) and non-systemically treated eyes (B). Statistical analysis was conducted using the Wilcoxon test $\left({ }^{\star} \mathrm{P}<0.05,{ }^{\star \star} \mathrm{P}<0.001,{ }^{\star \star \star} \mathrm{P}<0.001{ }^{\star \star \star \star} \mathrm{P}<0.0001\right.$, vs. baseline).

TABLE 2 | Inflammatory mediators in patients with or without systemic treatment at baseline.

\begin{tabular}{|c|c|c|c|}
\hline Mediator, mean \pm SD & No systemic treatment & Systemic treatment & P-value \\
\hline n (eyes/patients) & $20 / 20$ & $40 / 40$ & \\
\hline $\mathrm{TNF}-\alpha(\mathrm{pg} / \mathrm{mL})$ & $8.83 \pm 6.17$ & $9.39 \pm 10.44$ & 0.551 \\
\hline IL-1 $\beta$ (pg/mL) & $0.91 \pm 1.69$ & $0.46 \pm 0.52$ & 0.432 \\
\hline IL-6 (pg/mL) & $3.10 \pm 5.86$ & $6.44 \pm 16.43$ & 0.545 \\
\hline IL-8 (pg/mL) & $13.66 \pm 21.02$ & $12.70 \pm 11.57$ & 0.960 \\
\hline IL-10 (pg/mL) & $2.01 \pm 6.52$ & $0.52 \pm 0.97$ & 0.276 \\
\hline IL-17 (pg/mL) & $10.7 \pm 19.91$ & $15.48 \pm 30.68$ & 0.778 \\
\hline MCP-1 (pg/mL) & $617.41 \pm 404.47$ & $676.45 \pm 409.43$ & 0.846 \\
\hline VEGF (pg/mL) & $275.58 \pm 216.92$ & $226.82 \pm 155.29$ & 0.389 \\
\hline Tregs (\% CD3 $\left.{ }^{+} \mathrm{CD}^{+} \mathrm{PoxP}^{+}\right)$ & $3.78 \pm 2.46$ & $4.53 \pm 2.76$ & 0.392 \\
\hline
\end{tabular}

TNF, tumor necrosis factor; IL, interleukin; MCP, monocyte chemoattractant protein; VEGF, vascular endothelial growth factor; Tregs, regulatory T lymphocytes.

(OR: 0.67, 95\% CI: 0.45-1.00); $\mathrm{P}=0.042$ ). In contrast, higher baseline levels of Tregs were associated with UME sustained anatomical improvement (OR: 1.25, 95\% CI: 1.12-2.56, $\mathrm{P}=$ 0.049 ). The final model showed good goodness-of-fit (Lemeshow $\mathrm{P}=0.13$ ) (Figure 2). Given the observed associations, the inverse relationship between Tregs and IL-6 was evaluated. Spearman correlation showed no linear association between baseline levels of Tregs and IL-6 $(r=0.254, \mathrm{P}=0.109)$.

\section{DISCUSSION}

In this prospective study, we evaluated the association of several inflammatory cytokines and Tregs with anatomical improvement of UME. We analyzed the systemic levels of Tregs and eight serum immune mediators in a large group of patients who presented with macular edema secondary to noninfectious uveitis. Our findings show that high baseline serum levels of IL-6 were associated with poorer anatomical response in UME, whereas high levels of circulating Tregs were associated with UME sustained resolution throughout 12-month follow up.

The involvement of Tregs in controlling inflammation has gained considerable attention in uveitis as well as in other systemic autoimmune conditions $(15,16)$. To our knowledge, this is the first study that describes the association of Tregs with anatomical outcome in UME. Previous studies have compared Tregs levels between active uveitis, inactive uveitis and healthy control subjects. Some studies reported decreased levels of Tregs in active uveitis compared to inactive or quiescent status $(9,17)$ or healthy subjects (18), while others observed no differences (19) or even increased levels. Indeed, a previous study by our group showed no differences in Tregs levels between patients with active non-infectious uveitis and healthy subjects (20). Regarding the potential association of UME and Tregs levels, Yeh et al. did not observe differences between uveitis patients with and without UME (9). These discrepancies between studies could partly be attributed to the heterogeneity of uveitis subsets, clinical immunophenotyping and to the different strategies used to characterize Tregs. FoxP3 is the canonical transcription factor for naturally-occurring Tregs and is enriched in human $\mathrm{CD} 4{ }^{+} \mathrm{CD} 25^{\text {hi }} \mathrm{T}$ cells. Nevertheless, the $\mathrm{CD} 4^{+} \mathrm{CD} 25^{\text {hi }}$ population does not necessarily capture all $\mathrm{FoxP}^{+}$cells, therefore we defined $\mathrm{CD}^{+} \mathrm{CD}^{+}{ }^{+} \mathrm{FoxP}^{+}$as Tregs. The existence of several subtypes of Tregs involved in the modulation of the inflammatory response, could also explain such discrepancies. Thymus originated tTregs inhibit $\mathrm{T}$ effector cell trafficking, whereas inducible iTregs primarily prevent $\mathrm{T}$ cell priming by acting on antigen-presenting dendritic cells (21). The expression 
TABLE 3 | Clinical variables in UME with and without anatomical improvement.

\begin{tabular}{|c|c|c|c|c|c|}
\hline Clinical variables at baseline & & Total & No improvement & Improvement $^{1}$ & P-value $^{2}$ \\
\hline Total (eyes/patients) & & $60 / 60$ & $38 / 38$ & $22 / 22$ & \\
\hline \multicolumn{6}{|l|}{ Clinical imputs } \\
\hline Age (years, mean $\pm S D$ ) & & $51.1 \pm 15.1$ & $54 \pm 14.7$ & $46.1 \pm 14.8$ & 0.063 \\
\hline Female, $\mathrm{n}(\%)$ & & $35(58.3)$ & $24(63.2)$ & $11(50)$ & 0.319 \\
\hline Evolution time (months, mean $\pm \mathrm{SD}$ ) & & $17.3 \pm 31.5$ & $12.5 \pm 22.49$ & $25.7 \pm 42.2$ & 0.357 \\
\hline Bilateral, n (\%) & & $23(38.3)$ & $14(36.8)$ & $9(40.9)$ & 0.755 \\
\hline ACC (SUN) > 0.5+, n (\%) & & 29 (48.3) & $17(44.7)$ & $12(54.5)$ & 0.464 \\
\hline \multirow[t]{2}{*}{ Keratic precipitates, n (\%) } & Fines & $10(16.9)$ & 7 (18.9) & $3(13.6)$ & 0.725 \\
\hline & Granulomatous & $6(10.2)$ & $3(8.1)$ & $3(13.6)$ & \\
\hline Vitreous haze (NEI) > 0.5+, n (\%) & & $29(48.3)$ & $20(52.6)$ & $9(40.9)$ & 0.381 \\
\hline Chorioretinal lesions (any), (\%) & & $15(25)$ & $12(31.6)$ & $3(13.6)$ & 0.122 \\
\hline Anterior uveitis (SUN), n (\%) & & $16(26.7)$ & $9(23.7)$ & 7 (31.8) & 0.492 \\
\hline Unclassifiable uveitis, n (\%) & & 7 (20.6) & $5(23.8)$ & $2(15.4)$ & 0.654 \\
\hline \multirow[t]{2}{*}{ Ocular comorbidities, n (\%) } & Cataract & $16(26.7)$ & $8(21.1)$ & $8(36.4)$ & 0.196 \\
\hline & Glaucoma & $11(18.3)$ & $7(18.4)$ & 4 (18.2) & 0.982 \\
\hline \multirow[t]{3}{*}{ Treatments for UME, n (\%) } & None & $4(6.9)$ & $4(10.8)$ & $0(0)$ & \\
\hline & Local only & $14(23.3)$ & $10(27)$ & $4(19)$ & 0.191 \\
\hline & Systemic & $40(66.7)$ & $23(62.2)$ & $17(81)$ & \\
\hline \multicolumn{6}{|l|}{ SD-OCT imputs } \\
\hline Macular volume (mm3, mean $\pm \mathrm{SD})$ & & $11.9 \pm 1.9$ & $11.5 \pm 1.3$ & $12.6 \pm 2.6$ & 0.063 \\
\hline Thickened vitreo-macular interface, n (\%) & & 18(30) & $14(36.8)$ & 4 (18.2) & 0.129 \\
\hline Unaltered ellipsoid layer, n (\%) & & $35(58.3)$ & $25(65.8)$ & $10(45.5)$ & 0.124 \\
\hline Significant Tractional component, n (\%) & & $7(11.7)$ & $7(18.4)$ & $0(0)$ & 0.032 \\
\hline UME cystoid component, n (\%) & & 47 (78.3) & $29(76.3)$ & $18(81.8)$ & 0.618 \\
\hline Diameter of major cyst $(\mu \mathrm{m}$, mean $\pm \mathrm{SD})$ & & $330.1 \pm 475.5$ & $266.8 \pm 208.8$ & $433.5 \pm 723.2$ & 0.706 \\
\hline UME diffuse component, n (\%) & & $14(23.3)$ & $11(28.9)$ & $3(13.6)$ & 0.177 \\
\hline SRF transverse extent $(\mu \mathrm{m}$, mean $\pm \mathrm{SD})$ & & $363.1 \pm 520.2$ & $169 \pm 271.6$ & $621.9 \pm 665.8$ & 0.021 \\
\hline \multicolumn{5}{|l|}{ Immune imputs } & 0.137 \\
\hline Tregs $\left(\% \mathrm{CD}^{+} \mathrm{CD}^{+} \mathrm{PoxP}^{+}\right)$ & & $4.2 \pm 2.6$ & $4.0 \pm 2.3$ & $4.9 \pm 2.9$ & 0.153 \\
\hline $\mathrm{TNF} \alpha(\mathrm{pg} / \mathrm{mL})$ & & $8.9 \pm 8.2$ & $10.1 \pm 9.3$ & $7.1 \pm 5.9$ & 0.136 \\
\hline $\mathrm{IL}-1 \beta(\mathrm{pg} / \mathrm{mL})$ & & $0.7 \pm 1.4$ & $1.0 \pm 1.7$ & $0.3 \pm 0.3$ & 0.075 \\
\hline IL-6 (pg/mL) & & $4.7 \pm 11.9$ & $6.2 \pm 14.2$ & $2.3 \pm 6.7$ & 0.064 \\
\hline IL-8 (pg/mL) & & $12.9 \pm 18.5$ & $14.7 \pm 21.8$ & $10.0 \pm 11.1$ & 0.281 \\
\hline IL-10 (pg/mL) & & $1.5 \pm 5.4$ & $1.1 \pm 2.4$ & $2.1 \pm 8.3$ & 0.281 \\
\hline $\mathrm{IL}-17(\mathrm{pg} / \mathrm{mL})$ & & $12.7 \pm 25.4$ & $9.5 \pm 14.3$ & $17.9 \pm 36.9$ & 0.589 \\
\hline MCP-1 (pg/mL) & & $645.6 \pm 396.3$ & $656.3 \pm 410.8$ & $628.4 \pm 381.0$ & 0.822 \\
\hline VEGF (pg/mL) & & $263.8 \pm 195.8$ & $282.5 \pm 178.8$ & $233.6 \pm 221.7$ & 0.127 \\
\hline
\end{tabular}

${ }^{1}$ Improvement, defined as per decrease $\geq 20 \%$ or reach $<300 \mu \mathrm{m}$ in central subfield thickness at 6 months and maintained through month 12 .

${ }^{2}$ Chi-square or Fisher (categorical), Mann-Whitney (continuous).

ACC, anterior chamber cells; SUN standardization of uveitis nomenclature; NEI, National Eye Institute scale; UME, uveitic macular edema; SD-OCT, spectral-domain optical coherence tomography; SRF, subretinal fluid; TNF, tumor necrosis factor; IL, interleukin; MCP, monocyte chemoattractant protein; VEGF, vascular endothelial growth factor; Tregs, regulatory T cells.

of the transcription factor Helios is a useful marker for the identification of stable tTregs. However, we did not include Helios in our flow cytometry panel to characterize Tregs subtype. Previous studies determined Tregs levels in patients with and without UME in a cross-sectional manner, whereas the present study is prospective in nature, with the aim to investigate the predictive value of Tregs levels in the anatomical response after 12 months.

The differences observed in Tregs levels according to the anatomical event of UME improvement suggest that Tregs could be associated with the protective processes underlying UME resolution. In line with our findings, preclinical evidence shows that in experimental autoimmune uveoretinitis (EAU), the adoptive transfer of Tregs appears to confer protection from uveitis induced by the uveitogenic retinal antigen interphotoreceptor binding protein (IRBP) (22), and another study showed that retinal antigen-specific Foxp3+ Tregs contribute to the natural resolution of EAU and the maintenance of remission (23). Interestingly,
Gilbert et al. recently showed that clinical remission of noninfectious uveitis is characterized by an upregulation of peripheral Tregs polarized toward TIGIT and T-bet (24). TIGIT is a coinhibitory molecule expressed by Tregs. TIGIT $^{+}$Tregs seem to selectively inhibit Th17 and Th1, but not Th2 responses (25). Since it is known that the Th17 and Th1 subsets are pivotal in the pathogenesis of autoimmune disorders, TIGIT could be a biomarker of stable, functionally suppressive Tregs in such disorders. Even though we did not specifically analyze the suppressive function of Tregs, we observed an independent association between increased Tregs and anatomical improvement or resolution of UME, which was a $25 \%$ more frequently found for each unitary increase in Tregs log levels. Almost $67 \%$ of recruited patients received systemic treatment showing a good clinical ocular response, supporting the fact that systemic peripheral blood Tregs and their associated microenvironment influence ocular immunity and that, therefore, peripheral blood biomarkers can be useful in predicting ocular outcomes. 


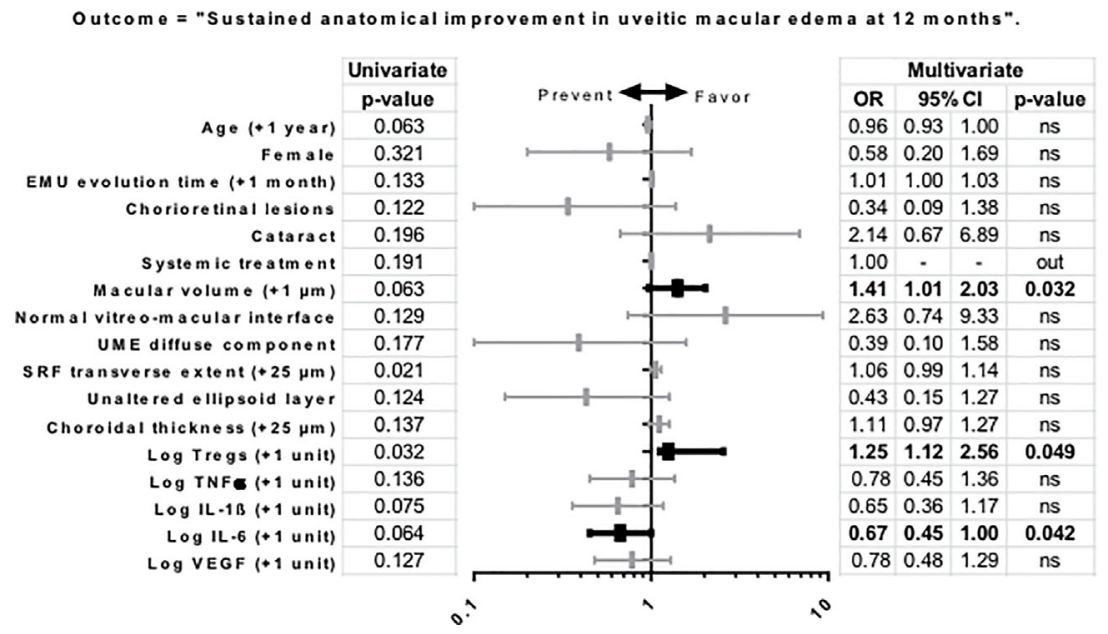

FIGURE 2 | Uni and multivariate regression model of variables as predictors of anatomic outcome in uveitic macular edema. Outcome: "sustained anatomical improvement", defined as per decrease $\geq 20 \%$ or reaches $<300 \mu \mathrm{m}$ in central subfoveal thickness at 6 months and maintained through month 12 . All covariates $(P<0.20)$ included in the multivariable model are shown. Quantitative variables were transformed to log scale. Statistically significant associations $(P$-value $<0.05)$ are noted in bold. Hosmer-Lemeshow goodness-of-fit test, $\mathrm{P}=0.13$. Abbreviations: $\mathrm{Cl}$, confidence interval; OR, odds ratio; UME, uveitic macular edema; SRF, subretinal fluid; TNF, tumor necrosis factor; IL, interleukin; VEGF, vascular endothelial growth factor; Tregs, regulatory $T$ lymphocytes.

On the other hand, the cytokine milieu in non-infectious uveitis has been extensively investigated (26-30). In the present study our focus was investigating the relationship between serum cytokines and anatomical improvement of UME. We therefore selected defined outcomes that may influence UME prognosis and can be easily and objectively assessed. BCVA, although a major clinical end result of UME, is not one of these features, since it can be easily biased by other parameters such as UME duration, presence of cataract, retinal scarring, or macular nonperfusion. We investigated whether anatomical improvement of UME could be predicted by immunological variables besides Tregs after adjusting for covariates in the model. The multivariate model showed that higher baseline levels of IL-6 were associated with lower CST improvement, which was a $33 \%$ less frequent for each unitary increase in IL-6 log-levels. IL-6 is a cytokine produced by several immune cells, in response to molecular patterns and affects multiple inflammatory cells and pathways. IL-6 is responsible for the induction of acute-phase proteins, differentiation of adaptive $\mathrm{T}$ cell responses, trafficking of acute and chronic inflammatory cells, regulation of homeostasis, and tissue regeneration (31). In the eye, significant elevation of IL-6 has been observed in ocular fluids (vitreous and aqueous) derived from retinal vein occlusion, diabetic macular edema, and refractory/chronic uveitis patients (32-34). Our group and others have previously reported that in patients with UME refractory to conventional therapies, systemic inhibition of IL-6 signaling with the IL-6 receptor (IL-6R) monoclonal antibody tocilizumab (TCZ-Actemra; Hoffmann-La Roche Ltd, Basel, Switzerland) was beneficial at 6,12 , and 24 months $(35,36)$, in particular with regards to achieving a reduction in CST. Indeed, IL-6 signaling blockade suppresses EAU (37), and it has been recently described that IL- 6 reversibly disrupts the integrity of the blood-retinal barrier in vitro (38), a key feature of the pathophysiology of macular edema. Our current study actually reinforces the concept of targeting systemic IL- 6 in UME, as patients with higher baseline serum levels of IL- 6 showed a less favorable anatomical response.

Despite the association of increased Tregs and reduced IL-6 levels with UME anatomical improvement, no linear association between IL-6 and Tregs was observed. The exact relationship between IL-6 and Tregs in modulating uveitis is not clearly understood. IL-6 is involved in the differentiation of $\mathrm{CD}^{+} \mathrm{T}$ cells into Th17 cells known to play a pivotal role in uveitis. Moreover, blockade of IL-6 signaling not only suppresses Th17 but also promotes Tregs in EAU (39). In other autoimmune disorders such as psoriasis, IL6 signaling prevents immune suppression by Tregs (40). Other authors have shown that IL6 overproduction in vivo inhibits inducible Treg generation from naïve $\mathrm{T}$ cells, but does not affect the development and function of natural Tregs (41). The inverse association of IL-6 and Tregs might occur only at the local site within the eye, but not in the systemic circulation, which could explain why we did not observe any association. Alternatively, the limited number of samples could also explain the lack of association. Thus, how IL6 modulates Treg function in the eye warrants further investigation.

Regarding the therapeutic approach, one may expect a relationship between systemic treatment and serum cytokine levels, as immunomodulatory agents are used to treat noninfectious uveitis. However, we did not observe significant differences in cytokine levels between none of the initial treatment modalities, either local, systemic, or a combination of both.

The main limitations of the present study include the sample size of eyes included in the analysis, the heterogeneity of uveitic 
conditions and immunomodulatory treatments, and the interindividual variability of cytokine levels. Despite these concerns, the strengths of the study include a prospective design, the use of standardized masked data collection protocols, a centralized center for the cytokine and Tregs determination, and the recruitment from multiple uveitis referral centers.

In conclusion, the results presented herein suggest that higher levels of Tregs may contribute to sustained anatomical improvement of UME, whereas increased systemic levels of IL6 may be a prognostic factor of poor anatomical outcome of UME. These findings could open the opportunity to more efficient and personalized therapeutic approaches to improve long-term visual prognosis in patients with macular edema secondary to non-infectious uveitis.

\section{DATA AVAILABILITY STATEMENT}

The raw data supporting the conclusions of this article will be made available by the authors, without undue reservation.

\section{ETHICS STATEMENT}

The studies involving human participants were reviewed and approved by Comite Ético de Investigación Clínica del Hospital

\section{REFERENCES}

1. Nussenblatt RB. The natural history of uveitis. Int Ophthalmol (1990) 14(56):303-8. doi: 10.1007/BF00163549

2. Suttorp-Schulten MSA, Rothova A. The possible impact of uveitis in blindness: A literature survey. Br J Ophthalmol (1996) 80(9):844-8. doi: 10.1136/bjo.80.9.844

3. Dick AD. Doyne lecture 2016: Intraocular health and the many faces of inflammation. Eye (2017) 31(1):87-96. doi: 10.1038/eye.2016.177

4. Munk MR, Kiss CG, Steiner I, Sulzbacher F, Roberts P, Kroh M, et al. Systematic correlation of morphologic alterations and retinal function in eyes with uveitis-associated cystoid macular oedema during development, resolution and relapse. Br J Ophthalmol (2013) 97:1289-96. doi: 10.1136/ bjophthalmol-2012-303052

5. Rothova A. Medical treatment of cystoid macular edema. Ocul Immunol Inflammation (2002) 10(4):239-46. doi: 10.1076/ocii.10.4.239.15589

6. Jeon S, Lee WK, Jung Y. Changes in the intraocular cytokine levels after intravitreal bevacizumab in uveitic macular edema. Ocul Immunol Inflammation (2012) 20(5):360-4. doi: 10.3109/09273948.2012.709576

7. Valencia X, Lipsky PE. CD4+CD25+FoxP3+regulatory T cells in autoimmune diseases. Nat Clin Pract Rheumatol (2007) 3(11):619-26. doi: 10.1038/ ncprheum0624

8. Ruggieri S, Frassanito MA, Dammacco R, Guerriero S. T reg lymphocytes in autoimmune uveitis. Ocul Immunol Inflammation (2012) 20(4):255-61. doi: 10.3109/09273948.2012.681830

9. Yeh S, Li Z, Forooghian F, Hwang FS, Cunningham MA, Pantanelli S, et al. CD4 +foxp3 + T-regulatory cells in noninfectious uveitis. Arch Ophthalmol (2009) 127(4):407-13. doi: 10.1001/archophthalmol.2009.32

10. Keino H, Takeuchi M, Usui Y, Hattori T, Yamakawa N, Kezuka T, et al. Supplementation of CD4+CD25+ regulatory $\mathrm{T}$ cells suppresses experimental autoimmune uveoretinitis. Br J Ophthalmol (2007) 91(1):105-10. doi: 10.1136/bjo.2006.099192
Clínic de Barcelona 2013/8574; Comité de Ética de la Investigación con Medicamentos de Euskadi, Hospital Universitario Cruces PI201406; and Comité Ético de Investigación Clínica del Hospital Clínico San Carlos de Madrid 13/244-E. The patients/ participants provided their written informed consent to participate in this study.

\section{AUTHOR CONTRIBUTIONS}

$\mathrm{VL}, \mathrm{MM}, \mathrm{AF}, \mathrm{DD}-\mathrm{V}, \mathrm{AA}$, and BM contributed to the design of the study and experiments. JM, VL, AF, DD-V, CE, JA, BB, MS, $\mathrm{AA}$, and $\mathrm{BM}$ performed the experiments, data capture, and analysis. JM, VL, MF-R, and BM performed the data interpretation. All authors contributed to the article and approved the submitted version.

\section{FUNDING}

This work was supported by the Ministry of Science and Innovation of Spain, 'Instituto de Salud Carlos III,' 'Fondo de Investigación Sanitaria' (PI13/00217, PI17/00316, and RD16/0008), and funds FEDER "Una manera de hacer Europa." We thank the support of the Generalitat of Catalunya (Secretaria d'Universitats i Recerca del Departament d'Economia i Coneixement de la Generalitat, 2017 SGR 0701.
11. Siepmann K, Biester S, Plšková J, Muckersie E, Duncan L, Forrester JV. CD4+CD25+ T regulatory cells induced by LPS-activated bone marrow dendritic cells suppress experimental autoimmune uveoretinitis in vivo. Graefe's Arch Clin Exp Ophthalmol (2007) 245(2):221-9. doi: 10.1007/ s00417-006-0356-9

12. Levin MH, Pistilli M, Daniel E, Gangaputra SS, Nussenblatt RB, Rosenbaum JT, et al. Incidence of visual improvement in uveitis cases with visual impairment caused by macular edema. Ophthalmology (2014) 121:58895.e1. doi: 10.1016/j.ophtha.2013.09.023

13. Ossewaarde-Van Norel A, Rothova A. Clinical review: Update on treatment of inflammatory macular edema. Ocul Immunol Inflammation (2011) 19(1):7583. doi: $10.3109 / 09273948.2010 .509530$

14. Jabs DA, Nussenblatt RB, Rosenbaum JT, Atmaca LS, Becker MD, Brezin AP, et al. Standardization of uveitis nomenclature for reporting clinical data. Results of the first international workshop. Am J Ophthalmol (2005) 140 (3):509-16. doi: 10.1016/j.ajo.2005.03.057

15. Rizzo LV, Xu H, Chan CC, Wiggert B, Caspi RR. IL-10 has a protective role in experimental autoimmune uveoretinitis. Int Immunol (1998) 10(6):807-14. doi: 10.1093/intimm/10.6.807

16. Roncarolo MG, Bacchetta R, Bordignon C, Narula S, Levings MK. Type $1 \mathrm{~T}$ regulatory cells. Immunol Rev (2001) 182:68-79. doi: 10.1034/j.1600065X.2001.1820105.x

17. Chen L, Yang P, Zhou H, He H, Ren X, Chi W, et al. Diminished frequency and function of CD4+CD25high regulatory $\mathrm{T}$ cells associated with active uveitis in Vogt-Koyanagi-Harada syndrome. Invest Ophthalmol Vis Sci (2008) 49(8):3475-82. doi: 10.1167/iovs.08-1793

18. Sugita S, Yamada Y, Kaneko S, Horie S, Mochizuki M. Induction of regulatory $\mathrm{T}$ cells by infliximab in behçet's disease. Invest Ophthalmol Vis Sci (2011) 52 (1):476-84. doi: 10.1167/iovs.10-5916

19. Commodaro AG, Peron JPS, Genre J, Arslanian C, Sanches L, Muccioli C, et al. IL-10 and TGF- $\beta$ immunoregulatory cytokines rather than natural regulatory $\mathrm{T}$ cells are associated with the resolution phase of Vogt-Koyanagi-Harada 
(VKH) syndrome. Scand J Immunol (2010) 72(1):31-7. doi: 10.1111/j.13653083.2010.02401.x

20. Molins B, Mesquida M, Lee RWJ, Llorenç V, Pelegrín L, Adán A. Regulatory T cell levels and cytokine production in active non-infectious uveitis: In-vitro effects of pharmacological treatment. Clin Exp Immunol (2015) 179(3):52938. doi: $10.1111 /$ cei.12479

21. Shevach EM, Thornton AM. tTregs, pTregs, and iTregs: Similarities and differences. Immunol Rev (2014) 259(1):88-102. doi: 10.1111/imr.12160

22. Silver PB, Agarwal RK, Su S-B, Suffia I, Grajewski RS, Luger D, et al. Hydrodynamic Vaccination with DNA Encoding an Immunologically Privileged Retinal Antigen Protects from Autoimmunity through Induction of Regulatory T Cells. J Immunol (2007) 179(8):5146-58. doi: 10.4049/ jimmunol.179.8.5146

23. Silver PB, Horai R, Chen J, Jittayasothorn Y, Chan C-C, Villasmil R, et al. Retina-Specific T Regulatory Cells Bring About Resolution and Maintain Remission of Autoimmune Uveitis. J Immunol (2015) 194(7):3011-9. doi: 10.4049/jimmunol.1501038

24. Gilbert RM, Zhang X, Sampson RD, Ehrenstein MR, Nguyen DX, Chaudhry M, et al. Clinical remission of sight-threatening non-infectious uveitis is characterized by an upregulation of peripheral T-regulatory cell polarized towards T-bet and TIGIT. Front Immunol (2018) 9:907. doi: 10.3389/ fimmu.2018.00907

25. Joller N, Lozano E, Burkett PR, Patel B, Xiao S, Zhu C, et al. Treg cells expressing the coinhibitory molecule TIGIT selectively inhibit proinflammatory Th1 and Th17 cell responses. Immunity (2014) 40(4):56981. doi: 10.1016/j.immuni.2014.02.012

26. Torun N, Callizo J, Orlic N, Scherer M, Hartmann C, Pleyer U. Serum cytokine receptor levels in noninfectious uveitis. Ophthalmic Res (2005) 37 (2):112-6. doi: 10.1159/000084271

27. Takase H, Futagami Y, Yoshida T, Kamoi K, Sugita S, Imai Y, et al. Cytokine profile in aqueous humor and sera of patients with infectious or noninfectious uveitis. Invest Ophthalmol Vis Sci (2006) 47(4):1557-61. doi: 10.1167/iovs.050836

28. Mesquida M, Molins B, Llorenç V, Sainz De La Maza M, Hernandez MV, Espinosa G, et al. Proinflammatory cytokines and c-reactive protein in uveitis associated with behçet's disease. Mediators Inflamm (2014) 2014:396204. doi: 10.1155/2014/396204

29. Sainz-de-la-Maza M, Molins B, Mesquida M, Llorenç V, Zarranz-Ventura J, Sala-Puigdollers A, et al. Interleukin-22 serum levels are elevated in active scleritis. Acta Ophthalmol (2016) 94(6):e395-9. doi: 10.1111/aos.13005

30. Molins B, Mesquida M, Llorenç V, Sainz de la Maza M, Adán A. Elevated Serum Immune Mediators and Subclinical Inflammation in HLA-A29-associated Birdshot Chorioretinopathy. Ocul Immunol Inflamm (2016) 24(6):647-652. doi: 10.3109/09273948.2015.1057601

31. Mesquida M, Molins B, Llorenç V, de la Maza MS, Adán A. Targeting interleukin-6 in autoimmune uveitis. Autoimmun Rev (2017) 16(10):1079-89. doi: 10.1016/j.autrev.2017.08.002

32. Owen LA, Hartnett ME. Soluble mediators of diabetic macular edema: the diagnostic role of aqueous VEGF and cytokine levels in diabetic macular edema. Curr Diabetes Rep (2013) 13:476-80. doi: 10.1007/s11892-0130382-z

33. Noma H, Funatsu H, Mimura T, Harino S, Hori S. Vitreous Levels of Interleukin-6 and Vascular Endothelial Growth Factor in Macular Edema with Central Retinal Vein Occlusion. Ophthalmology (2009) 116:87-93. doi: 10.1016/j.ophtha.2008.09.034

34. van Kooij B, Rothova A, Rijkers GT, de Groot-Mijnes JDF. Distinct cytokine and chemokine profiles in the aqueous of patients with uveitis and cystoid macular edema. Am J Ophthalmol (2006) 142:192-4. doi: 10.1016/j.ajo.2006.02.052

35. Mesquida M, Molins B, Llorenç V, Hernández MV, Espinosa G, Sainz de la Maza M, et al. Twenty-Four Month Follow-Up Of Tocilizumab Therapy For Refractory Uveitis-Related Macular Edema. Retina (2018) 38(7):1361-70. doi: 10.1097/IAE.0000000000001690

36. Mesquida M, Molins B, Llorenç V, Sainz de la Maza M, Adán A. Long-term effects of tocilizumab therapy for refractory uveitis-related macular edema. Ophthalmology (2014) 121:2380-6. doi: 10.1016/j.ophtha.2014.06.050

37. Rochfort KD, Collins LE, Murphy RP, Cummins PM. Downregulation of blood-brain barrier phenotype by proinflammatory cytokines involves NADPH oxidase-dependent ROS generation: Consequences for interendothelial adherens and tight junctions. PLoS One (2014) 9(7): e101815. doi: 10.1371/journal.pone.0101815

38. Mesquida M, Drawnel F, Lait PJ, Copland DA, Stimpson ML, Llorenç V, et al. Modelling macular edema: The effect of IL-6 and IL-6r blockade on human blood-retinal barrier integrity in vitro. Transl Vis Sci Technol (2019) 8(5):32. doi: $10.1167 /$ tvst.8.5.32

39. Hohki S, Ohguro N, Haruta H, Nakai K, Terabe F, Serada S, et al. Blockade of interleukin- 6 signaling suppresses experimental autoimmune uveoretinitis by the inhibition of inflammatory Th17 responses. Exp Eye Res (2010) 91:162-70. doi: 10.1016/j.exer.2010.04.009

40. Goodman WA, Levine AD, Massari JV, Sugiyama H, McCormick TS, Cooper KD. IL-6 Signaling in Psoriasis Prevents Immune Suppression by Regulatory T Cells. J Immunol (2009) 183(5):3170-6. doi: 10.4049/jimmunol.0803721

41. Fujimoto M, Nakano M, Terabe F, Kawahata H, Ohkawara T, Han Y, et al. The Influence of Excessive IL-6 Production In Vivo on the Development and Function of Foxp3 + Regulatory T Cells. J Immunol (2011) 186(1):32-40. doi: $10.4049 /$ jimmunol.0903314

Conflict of Interest: The authors declare that the research was conducted in the absence of any commercial or financial relationships that could be construed as a potential conflict of interest.

Copyright () 2020 Matas, Llorenç, Fonollosa, Diaz-Valle, Esquinas, de la Maza, Figueras-Roca, Artaraz, Berasategui, Mesquida, Adán and Molins. This is an open-access article distributed under the terms of the Creative Commons Attribution License (CC BY). The use, distribution or reproduction in other forums is permitted, provided the original author(s) and the copyright owner(s) are credited and that the original publication in this journal is cited, in accordance with accepted academic practice. No use, distribution or reproduction is permitted which does not comply with these terms. 